\title{
The presence of meniscal lesions is a strong predictor of neuropathic pain in symptomatic knee osteoarthritis: a cross-sectional pilot study
}

Camille Roubille ${ }^{1}$, Jean-Pierre Raynauld ${ }^{1}$, François Abram² ${ }^{2}$ Patrice Paiement ${ }^{2}$, Marc Dorais ${ }^{3}$, Philippe Delorme ${ }^{2}$, Louis Bessette ${ }^{4}$, André D Beaulieu ${ }^{5}$, Johanne Martel-Pelletier ${ }^{1}$ and Jean-Pierre Pelletier ${ }^{1 *}$

\begin{abstract}
Introduction: Pain in osteoarthritis (OA) has been classically attributed to joint structural damage. Disparity between the degree of radiographic structural damage and the severity of symptoms implies that factors other than the joint pathology itself contribute to the pain. Peripheral and central sensitization have been suggested as two of the underlying mechanisms that contribute to pain in OA. The aim of this study was to explore in symptomatic knee OA patients, the structural changes assessed by magnetic resonance imaging (MRI) that could be used as markers of neuropathic pain (NP).
\end{abstract}

Methods: This cross-sectional observational pilot study included 50 knee OA patients with moderate to severe pain (VAS $\geq 40$ ) in the target knee. The presence of NP was determined based on the PainDETECT questionnaire. Among the 50 patients included, 25 had PainDETECT score $\leq 12$ (unlikely NP), 9 had PainDETECT score between 13 and 18 (uncertain NP) and 16 had PainDETECT score $\geq 19$ (likely NP). WOMAC, PainDETECT, and VAS pain scores as well as knee MRI were assessed.

Results: Data showed no significant difference in demographic characteristics between the three groups. However, a positive and statistically significant association was found between the WOMAC pain $(P<0.001)$, function $(P<0.001)$, stiffness $(P=0.007)$ and total $(P<0.001)$ scores as well as higher VAS pain score $(P=0.023)$, and PainDETECT scores. Although no difference was found in the cartilage volume between groups, the presence of meniscal extrusion in both medial $(P=0.006)$ and lateral $(P=0.023)$ compartments, and presence of meniscal tears in the lateral compartment $(P=0.011)$, were significantly associated with increasing PainDETECT score. Moreover, the presence of bone marrow lesions in the lateral plateau and the extent of the synovial membrane thickness in the lateral recess were associated with increasing PainDETECT scores $(P=0.032, P=0.027$, respectively).

Conclusions: In this study, meniscal lesions, particularly extrusion, were found to be among the strongest risk factors for NP in knee OA patients.

Trial registration: ClinicalTrials.gov NCT01733277. Registered 16 November 2012.

\footnotetext{
* Correspondence: dr@jppelletier.ca

'Osteoarthritis Research Unit, University of Montreal Hospital Research Centre (CRCHUM), 900 rue Saint-Denis, Pavillon R, Suite R11.412, Montreal, QC,

Canada, H2X OA9

Full list of author information is available at the end of the article
} 


\section{Introduction}

Pain in osteoarthritis (OA) has been classically attributed to joint structural damage, and nearly all therapeutic strategies have been aimed at treating the pain derived from the joint. However, disparity between the degree of radiographic structural damage and the severity of symptoms, such as pain and functional limitations in OA patients, implies that factors other than the joint pathology itself, also contribute to the pain. Peripheral and central sensitizations have been suggested as two of the underlying mechanisms of pain in OA. Indeed, OA patients have been found to experience both nociceptive and neuropathic pain (NP) to varying degrees [1-5]. The peripheral nociceptors may be sensitized by, for example, inflamed synovium and damaged subchondral bone [5]. Continuous and intense nociceptive input from the OA knee joint may drive central sensitization, which may arise from chronic nociceptor stimulation and subsequent modification of central paintransmitting neurons, and may be clinically associated with NP qualities [1,2]. The diagnosis of NP is clinical and based on medical history, physical examination, and ancillary tests [6]. One such test, the PainDETECT, is a patientreport questionnaire extensively validated for the diagnosis of NP in various chronic pain conditions [7-11]. Higher scores suggest the likelihood of NP while lower scores are suggestive of a nociceptive pain. Hence, an NP component is unlikely if the score is $\leq 12$, uncertain if the score is 13 to 18 , and likely if the score is $\geq 19$ [7].

This cross-sectional study thus aimed at investigating, in knee OA patients with moderate to severe pain (visual analog scale (VAS) $\geq 40 \mathrm{~mm}$ ), the structural changes assessed by magnetic resonance imaging (MRI) that could be used as markers of uncertain (PainDETECT >12) or likely (PainDETECT $\geq 19$ ) NP.

\section{Methods}

\section{Study population and design}

This study is a multicentre, cross-sectional, single-blinded observational pilot study. The presence of NP was determined based on use of the PainDETECT questionnaire (score 0 to 38) [7-11]. The classification method defines $\mathrm{NP}$ as unlikely (score $\leq 12$ ), uncertain (score 13 to 18 ), or likely $(\geq 19)$. In this study, a total of 50 knee OA patients with moderate to severe pain (VAS $\geq 40$ ) in the most painful knee were enrolled, 25 having PainDETECT score $\leq 12$, 9 having PainDETECT score between 13 and 18, and 16 having PainDETECT score $\geq 19$. Since this is a pilot study, the number of patients was arbitrarily determined at 50 , which represents 25 patients with unlikely NP and 25 patients with uncertain or likely NP, a number deemed sufficient to identify the knee OA structural changes that could be preferentially associated with NP.

Subjects aged 40 years and older, followed in ambulatory clinics, with a diagnosis of primary knee OA according to the American College of Rheumatology criteria, of Kellgren-Lawrence (KL) radiological grades 2 and 3 , and being symptomatic for at least 1 month out of the 3 months preceding the study, and VAS pain score while walking on a flat surface $\geq 40 \mathrm{~mm}$, were eligible to be included in the study.

Subjects were excluded from the study if they met any of the following criteria at the beginning of, or during, the study: other bone or articular diseases (antecedents and/or current signs), isolated knee $\mathrm{OA}$ in the lateral compartment only defined by joint space loss, surgery on the target knee, comorbidities that restrict knee function, having taken any investigational drug within 30 days or 5 half-lives (whichever is greater) prior to entering the study, inability to give informed consent, meeting any contra-indication related to MRI, having taken either corticosteroids (oral, injectable or intra-articular injection of the target knee during the 12 weeks preceding the study) or intra-articular injections of hyaluronic acid in the target knee during the 26 weeks preceding the study.

This study, registered at ClinicalTrials.gov (NCT01 733277), was approved by the ethics committee IRB Services (Institutional Review Board Services), Aurora, ON, Canada. Written informed consent was obtained from all participants.

\section{Study visit: clinical, biological and X-ray outcomes}

The clinical characteristics included demographic data, the Western Ontario and McMasters Universities Osteoarthritis Index (WOMAC) questionnaire [12] and the VAS for global knee pain $(0 \mathrm{~mm}=$ no pain, $100 \mathrm{~mm}=$ most severe pain) within the last week, and the PainDETECT questionnaire completed at the study visit. Blood tests for sedimentation rate and C-reactive protein (CRP) were done. Knee X-rays taken within the last 12 months were used to verify that the subject satisfied radiological criteria of inclusion (KL grades 2 and 3).

\section{MRI acquisition and determination of structural changes}

MRI of the target knee was performed no more than 21 days after the study visit. The MRI acquisitions were performed on Philips Achieva $3 \mathrm{~T}$ units (Philips HealthCare, Markham, ON, Canada). The MRI examination comprised the following sequences: sagittal proton density-weighted fast spin-echo sequence with fat suppression (PD-FSE) (TR/TE, 3,550/25 ms; flip angle, 90 degrees; slice thickness/gap, $3 / 0 \mathrm{~mm}$; excitation number, 1; matrix size, $348 \times 348 \mathrm{px}$; field of view, $140 \mathrm{~mm}$; resolution, 0.398/0.398 mm; receiver bandwidth, $192 \mathrm{~Hz} /$ pixel; phase direction, $\mathrm{S} / \mathrm{I}$ ); sagittal three-dimensional intermediate-weighted fast spin-echo sequence with fat suppression (VISTA-SPAIR) (TR/TE, 1,500/30 ms; flip angle, 90 degrees; slice thickness/gap, 0.6/0 mm; excitation number, 1; matrix size, $232 \times 232 \mathrm{px}$; field of view, 
$140 \mathrm{~mm}$; resolution, $0.3125 / 0.3125 \mathrm{~mm}$; receiver bandwidth, $380 \mathrm{~Hz} /$ pixel; phase direction, A/P); and axial T1-weighted gradient-echo sequence non-fat suppressed (In-Out Phase) (TR/TE, 450/3.45 to $4.60 \mathrm{~ms}$; slice thickness/gap, 3.0/0 mm; excitation number, 1; matrix size, $256 \times 256 \mathrm{px}$; field of view, $180 \mathrm{~mm}$; resolution, $0.25 /$ $0.25 \mathrm{~mm}$; phase direction, $\mathrm{L} / \mathrm{R})$.

The cartilage volume was measured using the VISTASPAIR sequence and values determined with ArthroLab's fully automated quantitative MRI (qMRI) system (ArthroLab Inc, Montreal, QC, Canada) as described [13]. Meniscal tears were assessed with a fluid-sensitive sequence (PD-FSE) as recommended [14,15] and scored as absence $(-)$ or presence $(+)$ of a tear detected in any of the three segments (anterior horn, body, posterior horn) of the meniscus. Meniscal extrusion was assessed using the VISTASPAIR sequence, which allows for a precise evaluation of the extent of the extrusion, and scored as absence (-) or presence $(+)$ of partial or complete extrusion detected in any of the three segments of the meniscus as described $[14,16]$. Presence of bone marrow lesions (BMLs) was assessed with the PD-FSE sequence and evaluated as described [17]. The synovial membrane thickness (mm) was measured with the In-Out Phase sequence and measured in four subregions (medial and lateral articular recess and medial and lateral border of the suprapatellar bursa) as described $[17,18]$. Of note, the measurement of the synovial membrane thickness according to this method relies on the presence of synovial fluid, as assessed with the PS-FSE sequence, to localize the membrane in the different regions of interest. Thus, absence of synovial fluid that occurs, especially for the medial suprapatellar bursa, accounts for some missing values. The synovial effusion volume was assessed in its entirety using ArthroLab's fully automated qMRI system as described [19]. The evaluation of the structural changes was done under blinded conditions of patient identification and clinical data.

\section{Statistical analysis}

This exploratory study aimed to correlate the knee OA structural changes as assessed by quantitative and semiquantitative MRI scoring systems (cartilage volume, meniscal extrusion, BMLs, synovitis, and synovial effusion) with the presence of NP, by comparing the three study groups stratified according to PainDETECT scores $(\leq 12,13$ to 18 , and $\geq 19)$. Comparison of the demographic characteristics of the three study groups was carried out using the Kruskal-Wallis test for continuous variables and the chi-square test (or Fisher's exact test) for categorical variables. Comparison of the clinical and MRI characteristics was carried out using the JonckheereTerpstra trend test for continuous variables and the Cochran-Armitage trend test for categorical variables. A $P$ value $\leq 0.05$ was considered statistically significant. All statistical analyses were done using SAS software, version 9.3 (SAS Institute Inc., Cary, NC, USA).

\section{Results}

\section{Demographics}

The demographic characteristics of the three groups did not differ (Table 1). No statistical difference was found between PainDETECT groups with regard to the KL scores (Table 1). None of the patients received treatment for NP such as tricyclic antidepressants (TCAs), selective serotonin and norepinephrine reuptake inhibitors (SSRIs and SNRIs, respectively) or anticonvulsants (for example calcium channel $\alpha 2 \delta$ ligands).

\section{WOMAC, VAS, and PainDETECT pain scores}

A significant association was found between the WOMAC pain $(P<0.001)$, function $(P<0.001)$, stiffness $(P=0.007)$ and total $(P<0.001)$ scores and higher VAS pain $(P=0.023)$ and PainDETECT scores (Table 1).

\section{Inflammatory biomarkers}

No association was found between the sedimentation rate, the CRP values, and the PainDETECT scores (Table 1).

\section{Knee structural changes assessed by MRI Cartilage}

No association was found between the cartilage volume and the PainDETECT scores (Table 2).

\section{Meniscus}

The presence of meniscal extrusion in both medial $(P=$ 0.006) and lateral $(P=0.023)$ compartments was found to be significantly associated with increasing PainDETECT scores (Table 2). The presence of meniscal tears in the lateral compartment $(P=0.011)$ was also significantly associated with PainDETECT scores (Table 2).

\section{Bone marrow lesions}

Whereas no association between the presence of BMLs and the PainDETECT scores was found for the medial compartment, the association was significant in the lateral plateau $(P=0.032$, Table 2$)$.

\section{Synovial membrane thickness/synovial effusion}

No association was found between the synovial membrane thickness or synovial effusion size and the PainDETECT scores, except for the synovial membrane thickness in the lateral recess $(P=0.027)$ (Table 2).

\section{Discussion}

The aim of this cross-sectional pilot study was to explore in symptomatic knee OA patients the relationship between structural changes assessed by MRI and the presence of NP. 
Table 1 Demographic and clinical characteristics of patients based on PainDETECT score

\begin{tabular}{|c|c|c|c|c|}
\hline & $\begin{array}{l}\text { PainDETECT } \leq 12 \\
\mathrm{n}=\mathbf{2 5}\end{array}$ & $\begin{array}{l}\text { PainDETECT 13-18 } \\
\mathrm{n}=9\end{array}$ & $\begin{array}{l}\text { PainDETECT } \geq 19 \\
n=16\end{array}$ & $P$ value \\
\hline \multicolumn{5}{|l|}{ Demographic and clinical } \\
\hline Age, years & $66 \pm 9$ & $63 \pm 9$ & $67 \pm 7$ & $0.392^{*}$ \\
\hline Female, n (\%) & $18(72 \%)$ & $5(56 \%)$ & $10(63 \%)$ & $0.629 \neq$ \\
\hline Weight, kg & $82.5 \pm 14.1$ & $81.0 \pm 10.6$ & $87.0 \pm 18.9$ & $0.587^{*}$ \\
\hline OA duration, years & $7.7 \pm 8.1$ & $7.4 \pm 6.6$ & $8.1 \pm 4.6$ & $0.528^{*}$ \\
\hline $\mathrm{BMI}, \mathrm{kg} / \mathrm{m}^{2}$ & $31 \pm 6$ & $29.9 \pm 3$ & $33 \pm 6$ & $0.558^{*}$ \\
\hline NSAID users, n (\%) & $3(12 \%)$ & $0(0 \%)$ & $5(31 \%)$ & 0.1149 \\
\hline PainDETECT & $8.1 \pm 2.6$ & $15.6 \pm 1.1$ & $24.2 \pm 4.2$ & $<0.001$ \\
\hline \multicolumn{5}{|l|}{ WOMAC } \\
\hline Pain (0-100) & $43 \pm 17$ & $50 \pm 13$ & $65 \pm 14$ & $<0.001$ \\
\hline Function (0-100) & $45 \pm 23$ & $52 \pm 15$ & $68 \pm 14$ & $<0.001$ \\
\hline Stiffness (0-100) & $51 \pm 24$ & $49 \pm 18$ & $69 \pm 16$ & 0.007 \\
\hline Total (0-100) & $45 \pm 21$ & $51 \pm 14$ & $68 \pm 13$ & $<0.001$ \\
\hline VAS pain (0-100 mm) & $62 \pm 14$ & $62 \pm 13$ & $69 \pm 13$ & 0.023 \\
\hline Kellgren-Lawrence score & & & & $0.068+\S$ \\
\hline 2 & $14(56 \%)$ & $5(56 \%)$ & $5(31 \%)$ & \\
\hline 3 & $11(44 \%)$ & $4(44 \%)$ & $11(69 \%)$ & \\
\hline \multicolumn{5}{|l|}{ Inflammatory biomarkers } \\
\hline Sedimentation rate, $\mathrm{mm}$ & $12.4 \pm 9.0$ & $12.6 \pm 5.5$ & $15.2 \pm 20.8$ & 0.406 \\
\hline CRP, mg/l & $4.3 \pm 2.9$ & $6.8 \pm 9.1$ & $5.0 \pm 4.7$ & 0.432 \\
\hline
\end{tabular}

In the studied population of symptomatic knee OA patients with moderate to severe pain level, data first indicate a positive relationship between the level of $\mathrm{OA}$ symptoms and NP, in which the WOMAC scores as well as the VAS pain score were associated with the PainDETECT scores. Importantly, data also showed a greater likelihood of NP in patients with meniscal extrusion and lateral meniscal tears. These thus suggest that knee OA patients with a neuropathic component of pain have more severe symptoms, which appear related to meniscal lesions, more specifically extrusion, and are reflected, to a certain extent, by a trend toward a greater consumption of non-steroidal anti-inflammatory drugs (NSAIDs).

A higher WOMAC pain score in OA patients with a neuropathic component of pain has been previously reported [4] and could possibly explain the greater level of central sensitization that may have occurred. There was, however, no evidence in the present study, based on the current MRI findings, of an association with a more severe disease, which is in agreement with the previous report [4]. Results of this study also concur with the report of Murphy et al. [20] to the effect that the level of centrally mediated symptoms was likely independently associated with the pain severity. One cannot exclude the fact that nociception may have also played a role in the genesis of local pain and peripheral hyperalgia in the OA knee joint as well as central sensitization related to prolonged neuronal discharges [21]. The combination of peripheral and central pain mechanisms may possibly explain, at least in part, the discrepancy reported between the severity of OA changes and the pain intensity.

The mechanisms underlying NP-like symptoms in OA are still poorly understood. OA pain likely includes both nociceptive and neuropathic components. It has been suggested that the local damage to innervation as well as other joint structures may cause damage to peripheral nerves [3]. In this context, the association found between the presence of NP and BMLs and synovitis (synovial membrane thickness) in the lateral compartment is also very interesting. Although BMLs have been associated with knee pain [22-24], no report so far has related those changes with NP. A definite study with a larger number of patients would provide a better understanding of the current finding as well as its true meaning. A similar comment applies to the observation regarding the increase in synovial membrane thickness in the lateral compartment, as it is believed that synovial inflammation could sensitize peripheral nociceptors [25]. Therefore, it would seem 
Table 2 Knee structural changes assessed by MRI in patients based on PainDETECT score

\begin{tabular}{|c|c|c|c|c|}
\hline & $\begin{array}{l}\text { OA/NP- PainDETECT } \leq 12 \\
n=25\end{array}$ & $\begin{array}{l}\text { OA/NP+ PainDETECT 13-18 } \\
n=9\end{array}$ & $\begin{array}{l}\text { OA/NP+ PainDETECT } \geq 19 \\
n=16\end{array}$ & $P$ value \\
\hline \multicolumn{5}{|l|}{ MRI cartilage volume $\left(\mathrm{mm}^{3}\right)$} \\
\hline Global knee & $12,439 \pm 2,451$ & $12,181 \pm 3,894$ & $12,280 \pm 2,414$ & 0.347 \\
\hline Femur & $8,872 \pm 1,799$ & $8,998 \pm 2,769$ & $8,784 \pm 1,950$ & 0.381 \\
\hline Condyle & $5,873 \pm 1,220$ & $5,678 \pm 1,784$ & $5,825 \pm 1,552$ & 0.288 \\
\hline Plateau & $3,567 \pm 907$ & $3,183 \pm 1,467$ & $3,497 \pm 727$ & 0.395 \\
\hline Medial compartment & $6,232 \pm 1,258$ & $5,919 \pm 2,098$ & $6,101 \pm 1,473$ & 0.333 \\
\hline Femur & $4,594 \pm 884$ & $4,602 \pm 1,451$ & $4,474 \pm 1,103$ & 0.288 \\
\hline Condyle & $3,094 \pm 541$ & $2,878 \pm 912$ & $3,043 \pm 915$ & 0.172 \\
\hline Plateau & $1,639 \pm 516$ & $1,317 \pm 791$ & $1,626 \pm 524$ & 0.424 \\
\hline Lateral compartment & $6,207 \pm 1,404$ & $6,207 \pm 1,404$ & $6,180 \pm 1,476$ & 0.300 \\
\hline Femur & $4,278 \pm 1,030$ & $4,397 \pm 1,415$ & $4,309 \pm 1,002$ & 0.445 \\
\hline Condyle & $2,779 \pm 768$ & $2,800 \pm 1,019$ & $2,782 \pm 798$ & 0.496 \\
\hline Plateau & $1,928 \pm 595$ & $1,865 \pm 766$ & $1,870 \pm 738$ & 0.720 \\
\hline \multicolumn{5}{|c|}{ Meniscal extrusion (presence) } \\
\hline Medial compartment & $7(28 \%)$ & $3(33 \%)$ & $11(69 \%)$ & $0.006+$ \\
\hline Lateral compartment & $1(4 \%)$ & $1(11 \%)$ & $4(25 \%)$ & $0.023+$ \\
\hline \multicolumn{5}{|l|}{ Meniscal tears (presence) } \\
\hline Medial compartment & $14(56 \%)$ & $5(56 \%)$ & $10(63 \%)$ & $0.347 \dagger$ \\
\hline Lateral compartment & $3(12 \%)$ & $1(11 \%)$ & $7(44 \%)$ & $0.011+$ \\
\hline \multicolumn{5}{|c|}{ Bone marrow lesions (presence) } \\
\hline Medial compartment & $13(54 \%)^{a}$ & $8(89 \%)$ & $10(63 \%)$ & $0.246 \dagger$ \\
\hline Condyle & $4(17 \%)^{\mathrm{a}}$ & $1(11 \%)$ & $3(19 \%)$ & $0.447+$ \\
\hline Plateau & $12(50 \%)^{a}$ & $7(78 \%)$ & $10(63 \%)$ & $0.186 \dagger$ \\
\hline Lateral compartment & $9(38 \%)^{a}$ & $5(56 \%)$ & $7(44 \%)$ & $0.321+$ \\
\hline Condyle & $1(4 \%)^{a}$ & $0(0 \%)$ & $1(6 \%)$ & $0.395+$ \\
\hline Plateau & $8(33 \%)^{a}$ & $5(56 \%)$ & $10(63 \%)$ & $0.032 \dagger$ \\
\hline \multicolumn{5}{|c|}{ Synovial membrane thickness (mm) } \\
\hline Global & $2.23 \pm 0.66$ & $2.32 \pm 0.51$ & $2.13 \pm 0.52$ & 0.427 \\
\hline Medial compartment & $2.27 \pm 1.28^{b}$ & $2.14 \pm 0.58$ & $1.97 \pm 0.42^{c}$ & 0.390 \\
\hline Medial recess & $2.02 \pm 0.96^{d}$ & $1.91 \pm 0.62$ & $1.93 \pm 0.82^{c}$ & 0.399 \\
\hline Medial suprapatellar bursa & $2.89 \pm 1.57^{e}$ & $2.73 \pm 1.00^{f}$ & $2.13 \pm 0.68^{e}$ & 0.111 \\
\hline Lateral compartment & $2.21 \pm 0.75$ & $2.47 \pm 0.68$ & $2.19 \pm 0.73$ & 0.402 \\
\hline Lateral recess & $1.90 \pm 0.64^{\mathrm{a}}$ & $2.07 \pm 0.76$ & $2.15 \pm 0.55^{c}$ & 0.027 \\
\hline Lateral suprapatellar bursa & $2.64 \pm 1.42^{b}$ & $2.96 \pm 1.15^{9}$ & $2.26 \pm 1.18$ & 0.228 \\
\hline Synovial effusion (ml) & $11.22 \pm 7.74$ & $14.40 \pm 10.3$ & $12.40 \pm 6.40$ & 0.202 \\
\hline
\end{tabular}

Results are shown as mean \pm standard deviation (SD) unless otherwise indicated. $P$ values were assessed using the Jonckheere-Terpstra test for trend, and the †Cochran-Armitage trend test. ${ }^{a}, \mathrm{n}=24 ;{ }^{\mathrm{b}}, \mathrm{n}=22 ;{ }^{\mathrm{c}}, \mathrm{n}=15 ;{ }^{\mathrm{d}}, \mathrm{n}=21 ;{ }^{\mathrm{e}}, \mathrm{n}=12 ;{ }^{\mathrm{f}}, \mathrm{n}=6{ }^{\mathrm{g}}{ }^{\mathrm{g}}, \mathrm{n}=8$. MRI, magnetic resonance imaging; OA, osteoarthritis; NP, neuropathic pain; $n$, number of participants.

appropriate and logical to pursue this avenue of research in the future.

The association of knee pain with meniscal extrusion assessed by MRI in OA patients was recently reported [26]. A few possible explanations for the relationship between meniscal extrusion and knee pain are as follows. First, the association between meniscal extrusion and BMLs, a known source of pain [26], could possibly be due to the loss of mechanical protection provided by the meniscus. Second, it could be, as recently reported [27], that the increased vascular penetration and sensory nerve densities in the OA medial meniscus 
suggest a potential role of meniscal sensory nerve growth in knee OA pain, and could have contributed to the neuropathic component of the pain. Another possible explanation may be the mechanical stretching of the joint capsule, a richly innervated tissue, by the bulging meniscus. Our finding of an association between $\mathrm{NP}$ and lateral meniscal tear is somewhat unexpected as literature indicates that meniscal tears are not usually associated with symptoms [28]. Perhaps this finding is due to the role of possible confounding factors that will have to be further explored in a comprehensive study with a larger number of patients.

It is noteworthy that very few data exist regarding the relationship between meniscal lesions and NP in knee $\mathrm{OA}$ and this is a promising field of future research. The present study is particularly interesting as it is the first to report a clear association of meniscal lesions, more specifically extrusion, with NP. The finding of the association between the presence of meniscal extrusion and the PainDETECT scores makes this structural alteration a definite marker of NP. This finding is clinically relevant for various reasons. First, it argues for a pathophysiological relationship between NP and meniscal extrusion in knee OA and supports the examination for meniscal extrusion in knee OA patients with NP. This suggests that in daily practice, the predominance of a neuropathic component in such patients should encourage physicians to consider the use of MRI to establish a proper diagnosis. Second, a diagnosis of meniscal extrusion may help to identify knee OA patients who are more susceptible to benefit from a treatment aimed at controlling their symptoms more specifically. There is hope that this 'personalized therapeutic management' would avoid the prolonged use of anti-inflammatory drugs or even narcotic analgesics, preventing potential side effects, and that the patients would have a better response to treatment. Future research might explore whether managing meniscal extrusion, for instance with arthroscopic meniscal repair or resection, in such patients with NP would be beneficial.

This pilot study has limitations, the first being that it was an observational study and not a randomized controlled trial, the second being the arbitrary determination of the sample and, third, the diagnosis of NP was based solely on the PainDETECT questionnaire. However, as no gold standard test for NP diagnosis in OA is yet recognized, this questionnaire was used, as in many previous OA studies $[4,29,30]$, since it provides the greatest level of confidence. The relatively small sample size may also be a limiting factor. Nonetheless, this MRI study provides strong insight into the relationship between NP and meniscal lesions in knee OA, especially in patients with higher PainDETECT scores, hence with likely NP.

\section{Conclusions}

In summary, the findings of this study show that in knee OA patients, meniscal lesions are a definite major risk factor for NP. BMLs and synovitis also seem to be, to a certain extent, associated with NP; however, a definite study is needed to fully address the question, as they may be contributing factors in association with others.

\begin{abstract}
Abbreviations
BMLs: bone marrow lesions; CRP: C-reactive protein; KL: Kellgren-Lawrence; MRI: magnetic resonance imaging; NP: neuropathic pain; NSAIDs: non-steroidal anti-inflammatory drugs; OA: osteoarthritis; qMRI: quantitative MRI; SNRIs: selective norepinephrine reuptake inhibitors; SSRIs: selective serotonin reuptake inhibitors; TCAs: tricyclic antidepressants; VAS: visual analog scale; WOMAC: Western Ontario and McMasters Universities Osteoarthritis Index.
\end{abstract}

\section{Competing interests}

JMP and JPP are shareholders in ArthroLab. JPR and MD are consultants for ArthroLab. FA, PP, and PD are employees of ArthroLab. None of the authors has non-financial competing interests.

\section{Authors' contributions}

CR participated in the acquisition, analysis and interpretation of the data and drafting of the manuscript. JPR was responsible for the analysis and interpretation of the data. FA and PP participated in the acquisition of the data. MD was involved in the statistical analysis and interpretation of the data. PD performed the statistical analysis for the study. LB and ADB participated in the acquisition of the data. JMP contributed to the study design, acquisition, analysis and interpretation of the data, and drafting of the manuscript. JPP conceived the study, participated in its design and coordination, was involved in the acquisition, analysis and interpretation of the data, and drafting of the manuscript. All authors revised, read, and approved the final manuscript and agree to be accountable for the accuracy and integrity of the work.

\section{Acknowledgements}

This study was supported by an unrestricted grant from Merck \& Co. Inc., Whitehouse Station, NJ, USA, and in part by the Chair in Osteoarthritis of the University of Montreal and the CHUM (University of Montreal Hospital Center) Foundation. The authors are grateful to the participants who took part in the study and to Virginia Wallis for her assistance with the manuscript preparation.

\section{Author details}

${ }^{1}$ Osteoarthritis Research Unit, University of Montreal Hospital Research Centre (CRCHUM), 900 rue Saint-Denis, Pavillon R, Suite R11.412, Montreal, QC, Canada, H2X 0A9. ${ }^{2}$ Medical Imaging Research \& Development, ArthroLab Inc, 1871 rue Sherbrooke Est, Montreal, QC, Canada, H2K 1B6. ${ }^{3}$ StatSciences Inc, 60 rue Sylvio Leduc, Notre-Dame de l'île-Perrot, QC, Canada, J7V 7P2. ${ }^{4}$ Groupe de Recherche en Rhumatologie et Maladies Osseuses, 1200 Avenue de Germain-des-Prés, Suite 100, Sainte-Foy, QC, Canada, G1V 3M7. ${ }^{5}$ Centre de rhumatologie St-Louis, 3165 chemin St-Louis, Suite 360, Sainte-Foy, QC G1W 4R4, Canada.

Received: 14 May 2014 Accepted: 9 December 2014

Published online: 14 December 2014

\section{References}

1. Hochman JR, Davis AM, Elkayam J, Gagliese L, Hawker GA: Neuropathic pain symptoms on the modified painDETECT correlate with signs of central sensitization in knee osteoarthritis. Osteoarthritis Cartilage 2013, 21:1236-1242.

2. Hochman JR, Gagliese L, Davis AM, Hawker GA: Neuropathic pain symptoms in a community knee OA cohort. Osteoarthritis Cartilage 2011, 19:647-654.

3. Mease PJ, Hanna S, Frakes EP, Altman RD: Pain mechanisms in osteoarthritis: understanding the role of central pain and current approaches to its treatment. J Rheumatol 2011, 38:1546-1551. 
4. Ohtori S, Orita S, Yamashita M, Ishikawa T, Ito T, Shigemura T, Nishiyama H, Konno S, Ohta H, Takaso M, Inoue G, Eguchi Y, Ochiai N, Kishida S, Kuniyoshi K, Aoki Y, Arai G, Miyagi M, Kamoda H, Suzkuki M, Nakamura J, Furuya T, Kubota G, Sakuma Y, Oikawa Y, Suzuki M, Sasho T, Nakagawa K, Toyone T, Takahashi K: Existence of a neuropathic pain component in patients with osteoarthritis of the knee. Yonsei Med J 2012, 53:801-805

5. Schaible HG: Mechanisms of chronic pain in osteoarthritis. Curr Rheumatol Rep 2012, 14:549-556.

6. Gilron IC, Watson PN, Cahill CM, Moulin DE: Neuropathic pain: a practical guide for the clinician. CMAJ 2006, 175:265-275.

7. Freynhagen R, Baron R, Gockel U, Tolle TR: painDETECT: a new screening questionnaire to identify neuropathic components in patients with back pain. Curr Med Res Opin 2006, 22:1911-1920.

8. Hallstrom H, Norrbrink C: Screening tools for neuropathic pain: can they be of use in individuals with spinal cord injury? Pain 2011, 152:772-779.

9. Beith ID, Kemp A, Kenyon J, Prout M, Chestnut TJ: Identifying neuropathic back and leg pain: a cross-sectional study. Pain 2011, 152:1511-1516.

10. Baron R, Tolle TR, Gockel U, Brosz M, Freynhagen R: A cross-sectional cohort survey in 2100 patients with painful diabetic neuropathy and postherpetic neuralgia: Differences in demographic data and sensory symptoms. Pain 2009, 146:34-40.

11. Jespersen A, Amris K, Bliddal H, Andersen S, Lavik B, Janssen H, Poulsen PB: Is neuropathic pain underdiagnosed in musculoskeletal pain conditions? The Danish PainDETECTive study. Curr Med Res Opin 2010, 26:2041-2045.

12. Bellamy N, Buchanan WW, Goldsmith CH, Campbell J, Stitt LW: Validation study of WOMAC: a health status instrument for measuring clinically important patient relevant outcomes to antirheumatic drug therapy in patients with osteoarthritis of the hip or knee. J Rheumatol 1988, 15:1833-1840.

13. Dodin P, Pelletier JP, Martel-Pelletier J, Abram F: Automatic human knee cartilage segmentation from 3D magnetic resonance images. IEEE Trans Biomed Eng 2010, 57:2699-2711.

14. Berthiaume MJ, Raynauld JP, Martel-Pelletier J, Labonté F, Beaudoin G, Bloch DA, Choquette D, Haraoui B, Altman RD, Hochberg M, Meyer JM, Cline GA, Pelletier JP: Meniscal tear and extrusion are strongly associated with the progression of knee osteoarthritis as assessed by quantitative magnetic resonance imaging. Ann Rheum Dis 2005, 64:556-563.

15. Hunter DJ, Guermazi A, Lo GH, Grainger AJ, Conaghan PG, Boudreau RM, Roemer FW: Evolution of semi-quantitative whole joint assessment of knee OA: MOAKS (MRI Osteoarthritis Knee Score). Osteoarthritis Cartilage 2011, 19:990-1002.

16. Raynauld JP, Martel-Pelletier J, Berthiaume MJ, Beaudoin G, Choquette D, Haraoui B, Tannenbaum H, Meyer JM, Beary JF, Cline GA, Pelletier JP: Long term evaluation of disease progression through the quantitative magnetic resonance imaging of symptomatic knee osteoarthritis patients: correlation with clinical symptoms and radiographic changes. Arthritis Res Ther 2006, 8:R21.

17. Wildi LM, Raynauld JP, Martel-Pelletier J, Beaulieu A, Bessette L, Morin F, Abram F, Dorais M, Pelletier JP: Chondroitin sulphate reduces both cartilage volume loss and bone marrow lesions in knee osteoarthritis patients starting as early as 6 months after initiation of therapy: a randomised, double-blind, placebo-controlled pilot study using MRI. Ann Rheum Dis 2011, 70:982-989.

18. Pelletier JP, Raynauld JP, Abram F, Haraoui B, Choquette D, Martel-Pelletier J: A new non-invasive method to assess synovitis severity in relation to symptoms and cartilage volume loss in knee osteoarthritis patients using MRI. Osteoarthritis Cartilage 2008, 16:S8-S13.

19. Li W, Abram F, Pelletier JP, Raynauld JP, Dorais M, D'Anjou MA, Martel-Pelletier J: Fully automated system for the quantification of human osteoarthritic knee joint effusion volume using magnetic resonance imaging. Arthritis Res Ther 2010, 12:R173.

20. Murphy SL, Lyden AK, Phillips K, Clauw DJ, Williams DA: Association between pain, radiographic severity, and centrally-mediated symptoms in women with knee osteoarthritis. Arthritis Care Res (Hoboken) 2011, 63:1543-1549.

21. Arendt-Nielsen L, Hoeck HC: Peripheral and central sensitisation in osteoarthritis: implications for treatment. Eur Musculoskelet Rev 2011, 6:158-161.

22. Felson DT, Chaisson CE, Hill CL, Totterman SM, Gale ME, Skinner KM, Kazis L, Gale DR: The association of bone marrow lesions with pain in knee osteoarthritis. Ann Intern Med 2001, 134:541-549.
23. Felson DT, Niu J, Guermazi A, Roemer F, Aliabadi P, Clancy M, Torner J, Lewis CE, Nevitt MC: Correlation of the development of knee pain with enlarging bone marrow lesions on magnetic resonance imaging. Arthritis Rheum 2007, 56:2986-2992.

24. Wildi LM, Raynauld JP, Martel-Pelletier J, Abram F, Dorais M, Pelletier JP: Relationship between bone marrow lesions, cartilage loss and pain in knee osteoarthritis: results from a randomised controlled clinical trial using MRI. Ann Rheum Dis 2010, 69:2118-2124.

25. Arendt-Nielsen $L$, Nie $H$, Laursen MB, Laursen BS, Madeleine $P$, Simonsen $O H$, Graven-Nielsen T: Sensitization in patients with painful knee osteoarthritis. Pain 2010, 149:573-581.

26. Englund M, Roemer FW, Hayashi D, Crema MD, Guermazi A: Meniscus pathology, osteoarthritis and the treatment controversy. Nat Rev Rheumatol 2012, 8:412-419.

27. Ashraf S, Wibberley H, Mapp PI, Hill R, Wilson D, Walsh DA: Increased vascular penetration and nerve growth in the meniscus: a potential source of pain in osteoarthritis. Ann Rheum Dis 2011, 70:523-529.

28. Wenger A, Englund M, Wirth W, Hudelmaier M, Kwoh K, Eckstein F, Investigators OAl: Relationship of 3D meniscal morphology and position with knee pain in subjects with knee osteoarthritis: a pilot study. Eur Radiol 2012, 22:211-220.

29. Hochman JR, French MR, Bermingham SL, Hawker GA: The nerve of osteoarthritis pain. Arthritis Care Res (Hoboken) 2010, 62:1019-1023.

30. Valdes AM, Suokas AK, Doherty SA, Jenkins W, Doherty M: History of knee surgery is associated with higher prevalence of neuropathic pain-like symptoms in patients with severe osteoarthritis of the knee. Semin Arthritis Rheum 2014, 43:588-592.

\section{Submit your next manuscript to BioMed Central and take full advantage of:}

- Convenient online submission

- Thorough peer review

- No space constraints or color figure charges

- Immediate publication on acceptance

- Inclusion in PubMed, CAS, Scopus and Google Scholar

- Research which is freely available for redistribution

Submit your manuscript at www.biomedcentral.com/submit
C Biomed Central 